\title{
Strates
}

STRATES Matériaux pour la recherche en sciences sociales

5 | 1990

Conjuguer stratégies et territoires?

\section{Plus stratégique que le territoire, tu meurs...}

\section{Yves Luginbuhl}

\section{(2) OpenEdition}

\section{Journals}

\section{Édition électronique}

URL : http://journals.openedition.org/strates/1421

DOI : $10.4000 /$ strates. 1421

ISSN : $1777-5442$

Éditeur

Laboratoire Ladyss

Édition imprimée

Date de publication : 31 décembre 1990

ISSN : 0768-8067

\section{Référence électronique}

Yves Luginbuhl, «Plus stratégique que le territoire, tu meurs... », Strates [En ligne], 5 | 1990, mis en ligne le 16 mars 2007, consulté le 08 septembre 2020. URL : http://journals.openedition.org/strates/ 1421 ; DOI : https://doi.org/10.4000/strates.1421

Ce document a été généré automatiquement le 8 septembre 2020

Tous droits réservés 


\title{
Plus stratégique que le territoire, tu meurs...
}

\author{
Yves Luginbuhl
}

1 Hors de toute réflexion approfondie, et d'une manière générale dans mes travaux, je n'ai utilisé le terme de stratégie que pour désigner l'ensemble des actions et comportements d'acteurs divers, destinés à consolider ou conquérir une position politique ; c'est-à-dire - s'il est possible de la définir ainsi - une stratégie de pouvoir.

2 La question posée - existe-t-il des stratégies territoriales?- demande donc une réflexion volontairement orientée dans le sens de l'interrogation, inhabituelle à ma pratique de recherche. C'est le sens de cette note et je crois que ce préalable méritait d'être précisé.

$3 \mathrm{Si}$, de prime abord, la réponse semble évidente et affirmative, à y bien regarder, elle soulève effectivement une série de problèmes liés à de multiples champs - sémantique, spatial, social, politique, etc. - et c'est en ce sens qu'elle est intéressante. La première interrogation est en effet d'ordre sémantique et revient à préciser les limites entre stratégie, tactique, action, comportement, etc. Très banalement, je repartirai de la définition admise par le dictionnaire (Robert) : conduite et réalisation par les meilleurs moyens d'une politique, dont la différence avec tactique : ensemble des moyens coordonnés pour parvenir à un résultat, ne me paraît pas à première vue évidente.

4 C'est à propos de mes travaux réalisés dans le Boischaut que j'ai donc mené cette réflexion sur la pertinence du caractère territorial des stratégies des acteurs de l'aménagement : dans quelle mesure les stratégies conduites par des acteurs ou groupes d'acteurs de changement spatial dans le Boischaut sont-elles territoriales?

5 Un premier groupe d'acteurs, agriculteurs autochtones ou migrants, tentent de procéder à des regroupements parcellaires de leurs exploitations. Dans le contexte général du refus du remembrement par la majorité des habitants, souvent soutenu par les maires qui voient dans leur adhésion au non-remembrement le moyen d'échapper à l'échec électoral, ces agriculteurs pratiquent la restructuration foncière à l'amiable, en évitant la procédure administrative et juridique classique. Ce groupe est constitué de quelques agriculteurs, 6 à 10 environ qui, par une connaissance mutuelle des terres de leurs exploitations, procèdent à des échanges de parcelles. La stratégie mise en place, 
répondent à un objectif de restructuration foncière, est conduite et réalisée selon une trajectoire qui évite l'obstacle de la procédure administrative élaborée par le pouvoir central: nous la nommerons, par analogie à la science militaire, stratégie de contournement. Est-elle cependant une stratégie territoriale?

Les moyens mis en œuvre permettent peut-être de répondre à cette seconde partie de la question: ces moyens sont constitués, de la part de ces agriculteurs, par la connaissance qu'ils ont en commun du groupe social concerné; ils savent qui est intéressé par l'échange parcellaire, et dans un espace délimité avec précision (la limite de la parcelle cadastrale). En ce sens, la stratégie mise en œuvre possède un caractère territorial affirmé. Elle est évidemment spatiale, puisqu'elle consiste à modifier l'espace et à le mettre en une forme visée, mais celui-ci étant connu et déterminé dans ses limites géographiques et sociales, elle est également territoriale.

7 À l'inverse, si l'on se penche sur les opposants à la restructuration foncière, c'est une autre stratégie qui est développée, qui pourrait prendre les qualificatifs de stratégie de blocage ou d'occupation ou encore d'attentisme s'il s'agit des élus (attendre une conjoncture différente). Son caractère territorial est lui aussi relativement fort, car cette stratégie s'applique à un espace délimité, cependant à une échelle différente, celle du pays ou de la petite région, ou, plus vraisemblablement, celle d'une sous-unité territoriale (la Vallée Noire dans le Boischaut, par exemple).

Une troisième attitude a pu être observée : il s'agit d'un élu municipal qui, conscient du refus prévisible du remembrement de la part de la majorité de la population communale, mais également de la nécessité de restructurer les exploitations agricoles, procède selon une stratégie qualifiée ici de stratégie des Horace. Elle consiste à réduire la difficulté présentée par l'obstacle en divisant le territoire communal en trois parties et en y appliquant la procédure de remembrement partie après partie. La première zone de la commune où le maire tente l'expérience du remembrement est celle où, selon sa connaissance des diverses positions des habitants, la procédure aura le plus de chances de pouvoir être appliquée. En outre, en étant particulièrement vigilant sur l'ampleur des travaux connexes, il compte se servir de cette première mise en œuvre de remembrement pour passer à la seconde étape puis à la troisième, en faisant l'hypothèse que les habitants et propriétaires de ces zones seront convaincus des faibles répercussions de l'opération sur l'aspect de la commune et sur le bien-fondé de la restructuration.

9 Le caractère territorial de cette stratégie des Horace semble donc ici assez clair, puisqu'elle est conduite sur un territoire délimité dans son ensemble et, qui plus est, dans ses sous-parties, et que les moyens mis en œuvre font intervenir la connaissance du territoire en question, tant du point de vue social que des points de vue économique et écologique. Il s'agit bien d'une stratégie développée afin de mettre en forme un territoire selon un objectif précis.

10 Il semble cependant que le caractère territorial de ces stratégies puisse être attribué au fait qu'elles concernent des actions sur le foncier, mais il n'est pas évident qu'il en soit de même pour des actions portant sur d'autres éléments de l'aménagement.

11 La question se pose effectivement dans le cas d'installation de firmes industrielles dans la même région. La première firme considérée, entreprise de fabrication de vêtements, s'est installée dans le canton de Saint-Sévère pour bénéficier de la présence d'une main-d'œuvre féminine à la recherche d'un emploi et peu revendicative, représentée par les femmes d'exploitants agricoles. Cette installation s'appuie donc sur la 
connaissance du milieu social de la région, certes, mais cette connaissance ne s'exprime pas dans un espace délimité. La stratégie serait donc avant tout spatiale et non pas territoriale. En ce sens, une différence se fait jour avec les stratégies des entreprises privées du secteur agroalimentaire évoquées par Françoise Plet $^{1}$; l'objectif de l'entrepreneur installé dans le Boischaut ne consiste pas à constituer un territoire cohérent au plan des localisations de la main-d'œuvre, car peu lui importe que ses employées résident dans telle ou telle partie de la région. L'essentiel est que leur profil socioprofessionnel soit conforme aux besoins de l'entreprise telle qu'il se la représente. Le territoire concerné par son entreprise est donc un espace mou, sans limites précises mais fluctuantes.

12 Le second cas analysé, celui d'une entreprise de produits pharmaceutiques, est plus problématique: la firme s'est installée à La Châtre pour les mêmes raisons que la première mais, en outre, elle a choisi cette implantation pour la qualité des eaux disponibles, nécessaires à son processus de fabrication. Le caractère de la stratégie d'installation de cette entreprise ne peut donc pas être qualifié de territorial fort, bien qu'il soit malgré tout un peu plus territorial que le premier, se fondant sur une spécificité régionale reconnue, la qualité de son eau.

D'autres cas pourraient être analysés, qu'il s'agisse de groupes d'agriculteurs ou de résidents non-agriculteurs ou encore de firmes industrielles, dont le caractère territorial des stratégies de développement, de pouvoir, etc. est variable.

En conclusion, on peut dire que ces stratégies ne sont pas uniquement et exclusivement territoriales, et qu'il existe différents types de stratégies que l'on pourrait peut-être classer selon divers critères: soit du point de vue de leur nature (stratégies de contournement, d'occupation, d'attentisme, des "Horace ", etc.), soit du point de vue de leur objectif (stratégies spatiales, de pouvoir, de protection, territoriales, de localisation, etc.). Il serait peut-être intéressant de proposer une sorte d'inventaire de ces types de stratégies.

En second lieu, la distinction entre stratégies territoriales et spatiales ne dépend peutêtre pas uniquement de la nature et des délais de réaction des acteurs ; interviendrait également la représentation de l'espace que se font les acteurs de sa dynamique, représentation dans laquelle s'organise une hiérarchie des caractères sociaux, écologiques, économiques en fonction de l'objectif de la stratégie. Il me semble qu'il y a là une autre piste de recherche à explorer.

\section{NOTES}

1. Cf. dans ce numéro de STRATES: PLET Françoise, « Stratégies territoriales : valeurs et usages dans les recherches rurales et agroalimentaires de STRATES ». 


\section{RÉSUMÉS}

L'analyse de l'évolution du paysage d'une petite région agricole, le Boischaut, a été l'occasion de se poser la question du caractère territorial des stratégies de différents acteurs locaux. Celles-ci peuvent en effet avoir un caractère stratégique plus ou moins marqué, mais on a identifié différents types de stratégies territoriales : de contournement, d'attentisme, des « Horace »... Les stratégies sont en tout cas plus territoriales lorsqu'elles concernent le foncier que d'autres domaines.

\section{Is there anything more strategic than a territory?}

Considering the evolution of the landscape in a rural area, the "Boischaut " (in the center of France), the strategies of local actors have been invesstigated to analyze their territorial contents. In fact, all local actors have more or less territorial concerns, but some types of territorial strategies can be identified, such as wait and see attitudes, bypass behaviours, etc. Anyhow, it is when agricultural land is at stake that territorial strategies are at their most.

\section{INDEX}

Mots-clés : Paysage, territoire, stratégie, Aménagement rural

Keywords : territory, landscape, strategy, Rural management

\section{AUTEUR}

\section{YVES LUGINBUHL}

Ingénieur agronome, chargé de recherche au CNRS, il s'est spécialisé depuis une vingtaine d'années sur les rapports entre l'aménagement de l'espace et les représentations du paysage. Il a publié en 1989 : Paysages - textes et représentations - du siècle des Lumières à nos jours, La Manufacture. 